\title{
Generalized combination complex synchronization of new hyperchaotic complex Lü-like systems
}

\author{
Cuimei Jiang and Shutang Liu*
}

"Correspondence: stliu@sdu.edu.cn College of Control Science and Engineering, Shandong University, Jinan, 250061, P.R. China

\section{严 Springer}

\begin{abstract}
In this paper, a new hyperchaotic complex system is presented and its dynamical properties are discussed by phase portraits, bifurcation diagrams, and the Lyapunov exponents spectra. Noticeably, based on two drive complex systems and one response complex system with different dimensions, we propose generalized combination complex synchronization and design a general controller. Additionally, we investigate generalized combination complex synchronization between real systems and complex systems via two complex scaling matrices. Two examples, which include two chaotic complex systems driving one new hyperchaotic complex system and two new hyperchaotic complex systems driving one chaotic real system, are shown to demonstrate the effectiveness and feasibility of the schemes.
\end{abstract}

Keywords: hyperchaotic complex systems; chaotic attractors; Lyapunov exponents; generalized combination complex synchronization

\section{Introduction}

In 1982, Fowler et al. [1] proposed the complex Lorenz equations, which is the pioneering work in the domain of complex systems. After that, chaotic and hyperchaotic complex systems have been extensively studied owing to their important applications in physical systems, image processing and in particular in secure communication [2-4]. And researchers presented many chaotic and hyperchaotic complex systems, such as the complex Lorenz system [5], the complex Chen system [6], the complex Lü system [6], the hyperchaotic complex Lorenz system [7], the hyperchaotic complex Lü system [8], and so on. Compared with chaotic systems, the behavior of hyperchaotic complex systems is more complex and richer. Hence, when applying the hyperchaotic complex systems to secure communication, it is better to increase the complexity and the security of the transmitted information.

On the other hand, with the development of complex systems, synchronization of chaotic complex systems has gained a great deal of attentions. Some synchronization schemes of chaotic real systems were extended to the complex space, such as complete synchronization [9], anti-synchronization [10, 11], projective synchronization [12], etc. Recently, many authors have studied some new kinds of synchronization for complex dynamical systems, for example, complex complete synchronization [13], complex projective synchronization [14], complex modified projective synchronization [15, 16], and so forth.

(c) 2015 Jiang and Liu; licensee Springer. This article is distributed under the terms of the Creative Commons Attribution 4.0 International License (http://creativecommons.org/licenses/by/4.0/), which permits unrestricted use, distribution, and reproduction in any medium, provided you give appropriate credit to the original author(s) and the source, provide a link to the Creative Commons license, and indicate if changes were made. 
Since complex variables increase the diversity and the security of the transmitted signals, these synchronization methods of chaotic complex systems have potential applications in secure communication and image processing.

However, most of the above-mentioned works mainly focus on the usual drive-response synchronization which has one drive system and one response system. To improve the ability of anti-attack and anti-translated of the transmitted information, Luo et al. [17] proposed combination synchronization which has two drive real systems and one response real system. Subsequently, Wu and Fu [18] studied increased-order and reduced-order combination synchronization in the real space concerning two specific examples. Soon afterwards, Zhou et al. [19] introduced combination synchronization to the complex space and carried out synchronization of three identical or different nonlinear complex hyperchaotic systems. Very recently, Sun et al. [20] investigated combination complex synchronization between two drive chaotic complex systems and one response chaotic complex system. These synchronization schemes occur in chaotic complex systems with the same dimensions.

To the best of our knowledge, there are few papers discussing combination synchronization among two drive systems and one response system with different dimensions in the complex space. As a matter of fact, for nonlinear systems with different dimensions, a lot of synchronization phenomena exist in reality, especially in the chemical and biological sciences. For instance, we can observe the physiological synchronization phenomena between higher-dimensional and lower-dimensional thalamic neurons as well as between the circulatory and respiratory systems [21]. Therefore, it is meaningful and valuable to study synchronization between two drive systems and one response system with different dimensions from the application point of view.

Inspired by the above discussion, we introduce a new hyperchaotic complex system to investigate generalized combination complex synchronization between two drive complex systems and one response complex system with different structures. Meanwhile, a general controller is designed to synchronize chaotic complex systems in the sense of generalized combination complex synchronization. By virtue of two complex scaling matrices, we establish a link between real chaos and complex chaos. The proposed generalized combination complex synchronization will contain complex projective synchronization, combination synchronization, and combination complex synchronization. Consequently, our work will extend the previous results.

The remainder of this paper is organized as follows. In Section 2, we present a hyperchaotic complex Lü-like system and study its dynamical properties including symmetry, equilibria and stability, Lyapunov exponents and fractal dimensions, as well as hyperchaotic attractors. Section 3 introduces generalized combination complex synchronization and designs a general controller. Two typical examples are treated to exhibit the effectiveness and correctness of the proposed methods. Finally, a concluding remark is given in Section 4.

\section{A new hyperchaotic complex Lü-like system}

In 2008, Zhou et al. [22] studied the Lü-like or Pan system which can be described as

$$
\left\{\begin{array}{l}
\dot{x}=a(y-x), \\
\dot{y}=c x-x z, \\
\dot{z}=x y-b z,
\end{array}\right.
$$




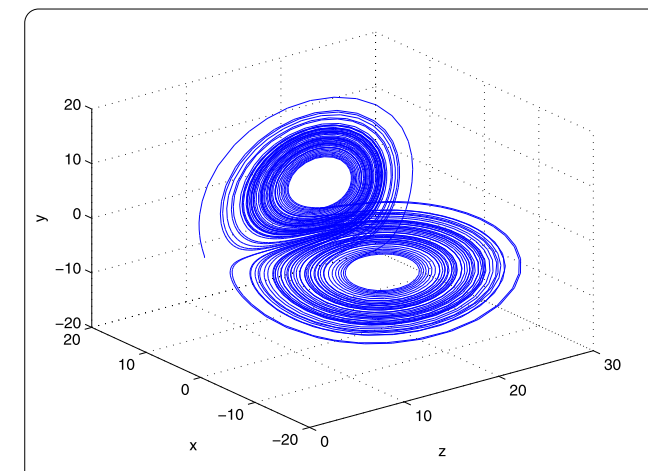

(a)

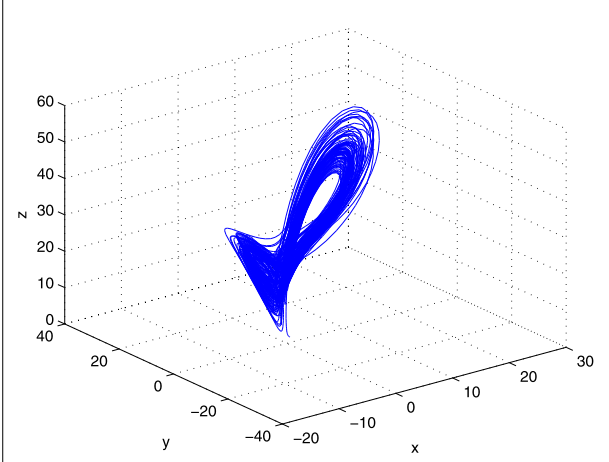

(c)

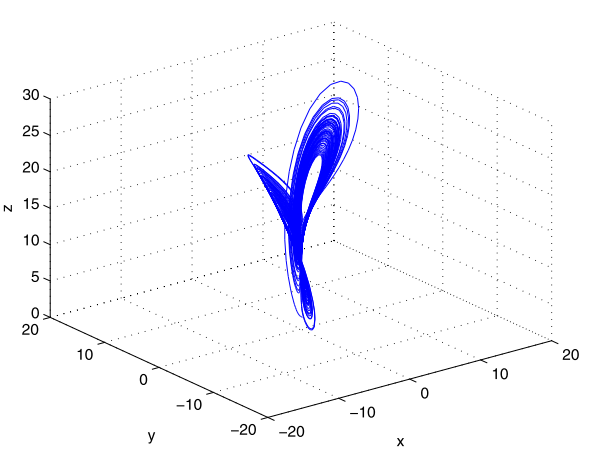

(b)

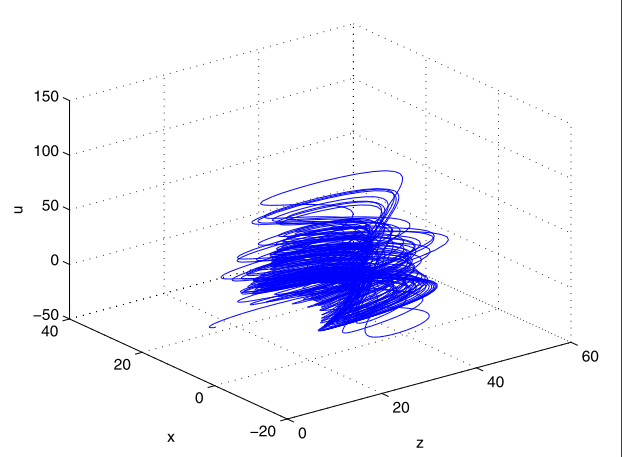

(d)

Figure 1 Attractors of chaotic system (1) and hyperchaotic system (2).

where $a, b$, and $c$ are real constants. When the parameters are chosen as $a=10, b=2$, and $c=16$, the Lü-like system (1) is chaotic as shown in Figure 1(a)-(b).

Recently, a new modified hyperchaotic Lü-like or Pan system [23] has been constructed by introducing a state feedback controller, which can be expressed by

$$
\left\{\begin{array}{l}
\dot{x}=a(y-x), \\
\dot{y}=c x-x z+u, \\
\dot{z}=x y-b z, \\
\dot{u}=-d y
\end{array}\right.
$$

where $a, b, c$, and $d$ are real constants, $(x, y, z, u)^{T}$ is a real state vector. The hyperchaotic attractors of system (2) are plotted in Figure 1(c)-(d) with $a=10, b=8 / 3, c=28$, and $d=10$. In this work, the complex extension of the modified hyperchaotic Lü-like system is firstly designed by

$$
\left\{\begin{array}{l}
\dot{x}_{1}=a\left(x_{2}-x_{1}\right), \\
\dot{x}_{2}=c x_{1}-x_{1} x_{3}+x_{4}, \\
\dot{x}_{3}=\frac{1}{2}\left(\bar{x}_{1} x_{2}+x_{1} \bar{x}_{2}\right)-b x_{3}, \\
\dot{x}_{4}=-\frac{d}{2}\left(\bar{x}_{2}+x_{2}\right),
\end{array}\right.
$$

where $a, b, c$, and $d$ are real positive parameters, $x_{1}=m_{1}+j m_{2}$ and $x_{2}=m_{3}+j m_{4}$ are complex variables, $x_{3}=m_{5}$ and $x_{4}=m_{6}$ are real variables. The hyperchaotic complex Lü- 
like system can be rewritten as follows:

$$
\left\{\begin{array}{l}
\dot{m}_{1}=a\left(m_{3}-m_{1}\right), \\
\dot{m}_{2}=a\left(m_{4}-m_{2}\right), \\
\dot{m}_{3}=c m_{1}-m_{1} m_{5}+m_{6}, \\
\dot{m}_{4}=c m_{2}-m_{2} m_{5}, \\
\dot{m}_{5}=m_{1} m_{3}+m_{2} m_{4}-b m_{5}, \\
\dot{m}_{6}=-d m_{3} .
\end{array}\right.
$$

In what follows, we investigate the basic dynamical properties of system (4).

\subsection{Symmetry and invariance}

Note that the symmetry of system (4): It is symmetric about the $m_{5}$-axis, which means it is invariant for the coordinate transformation of $\left(m_{1}, m_{2}, m_{3}, m_{4}, m_{5}, m_{6}\right) \rightarrow\left(-m_{1},-m_{2}\right.$, $\left.-m_{3},-m_{4}, m_{5},-m_{6}\right)$.

\subsection{Dissipation}

System (4) is dissipative under the condition $2 a+b>0$, since

$$
\nabla V=\sum_{k=1}^{6} \frac{\partial \dot{m}_{k}}{\partial m_{k}}=-2 a-b .
$$

\subsection{Equilibria and stability}

By solving the equations $\dot{m}_{1}=0, \dot{m}_{2}=0, \dot{m}_{3}=0, \dot{m}_{4}=0, \dot{m}_{5}=0$, and $\dot{m}_{6}=0$, we obtain three equilibrium points of system (4): $E_{1}=(0,0,0,0,0,0)$ and $E_{2,3}=(0, \pm \sqrt{b c}, 0, \pm \sqrt{b c}$, $0,0)$. To study the stability of the zero equilibrium point $E_{1}$, we have the Jacobian of system (4) at $E_{1}$ as follows:

$$
J_{E_{1}}=\left(\begin{array}{cccccc}
-a & 0 & a & 0 & 0 & 0 \\
0 & -a & 0 & a & 0 & 0 \\
c & 0 & 0 & 0 & 0 & 1 \\
0 & c & 0 & 0 & 0 & 0 \\
0 & 0 & 0 & 0 & -b & 0 \\
0 & 0 & -d & 0 & 0 & 0
\end{array}\right) .
$$

By a simple computation, the characteristic polynomial of the Jacobian matrix $J_{E_{1}}$ is obtained as

$$
(\lambda+b)\left(\lambda^{2}+a \lambda-a c\right)\left(\lambda^{3}+a \lambda^{2}-(a c-d) \lambda+a d\right)=0 .
$$

According to the Routh-Hurwitz theorem, we deduce that $E_{1}$ will be stable when $a>0$, $b>0, c<0$, and $d>0$. Otherwise, it is an unstable fixed point. Similarly, we can discuss the stability of the equilibrium points $E_{2}$ and $E_{3}$.

\subsection{Lyapunov exponents and fractal dimensions}

In the sequel, the Lyapunov exponents and fractal dimension of system (4) are calculated. By means of the Runge-Kutta method of order 4 in the MATLAB environment, we obtain the Lyapunov exponents for the case of $a=10, b=8 / 3, c=28$, and $d=10$ with the 
Figure 2 Lyapunov exponents spectrum of system (4) with $(a, b, c, d)=(10,8 / 3,28,10)$.

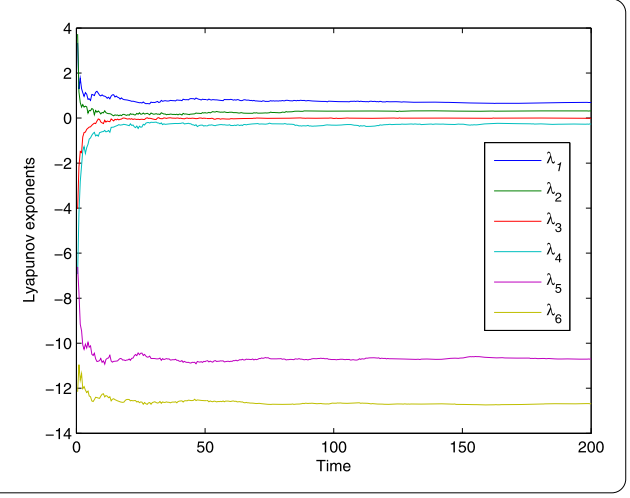

initial condition $x(0)=(1+j, 1+j, 2,3)$; see Figure 2. Here, the six Lyapunov exponents of system (4) are $\lambda_{1}=0.689209, \lambda_{2}=0.314361, \lambda_{3}=-0.009003 \approx 0, \lambda_{4}=-0.275494$, $\lambda_{5}=-10.697494$, and $\lambda_{6}=-12.685651$. Since $\lambda_{1}$ and $\lambda_{2}$ are positive values, system (4) is hyperchaotic for this choice of $a, b, c$, and $d$. Thus, we can calculate the fractal dimension [24] as follows:

$$
D=j+\frac{1}{\left|\lambda_{j+1}\right|} \sum_{i=1}^{j} \lambda_{i}=4+\frac{\lambda_{1}+\lambda_{2}+\lambda_{3}+\lambda_{4}}{\left|\lambda_{5}\right|}=4.06722 .
$$

\subsection{Hyperchaotic behavior and attractors}

System (4) is hyperchaotic when $(a, b, c, d)=(10,8 / 3,28,10)$ and $x(0)=(1+j, 1+j, 2,3)$. Figure 3 shows the hyperchaotic attractors of system (4) in different phase planes and projections.

Next, we calculate numerically the values of the parameters of (4) at which chaotic attractors exist under the above conditions. Now we consider the following two cases.

(i) Fix $b=8 / 3, c=28, d=10$, and let $a$ vary: To observe the Lyapunov exponents spectrum clearly, we plot the values of $\lambda_{1}, \lambda_{2}$, and $\lambda_{3}$ in Figure 4(a), while the values of $\lambda_{4}, \lambda_{5}$, and $\lambda_{6}$ are shown in Figure 4(b). From Figure 4(a), it is obvious that system (4) has hyperchaotic attractors for $a \in[3.3,27.4]$, while it has chaotic attractors when $a \in[1.2,3.2]$. The above results can be demonstrated by the bifurcation diagram which is displayed in Figure 5(a), while Figure 5(b)-(d) describe attractors of system (4).

(ii) Fix $a=10, c=28, d=10$, and let $b$ vary: The values of $\lambda_{1}, \lambda_{2}$, and $\lambda_{3}$ are plotted in Figure 6(a), while the values of $\lambda_{4}, \lambda_{5}$, and $\lambda_{6}$ are shown in Figure 6(b). From Figure 6(a), we see that system (4) has hyperchaotic attractors for $b \in[0.51,4.99]$, chaotic attractors for $b \in[0.18,0.36]$ and $[0.44,0.5]$, and solutions of system (4) that approach fixed points for $b \in[0.37,0.39]$. Corresponding bifurcation diagram with the step size of 0.1 is plotted in Figure 7(a). Meanwhile, the attractors of system (4) are depicted in Figure 7(b)-(c).

\section{Generalized combination complex synchronization}

The aim of this section is to present generalized combination complex synchronization and design a general controller. Then two simulation examples are given to verify the effectiveness of the schemes. 


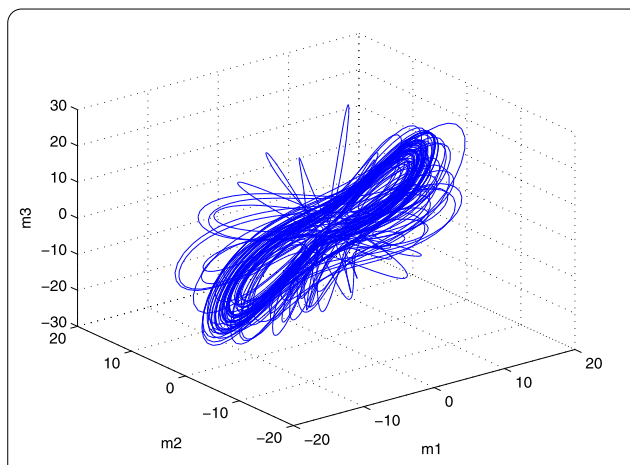

(a)

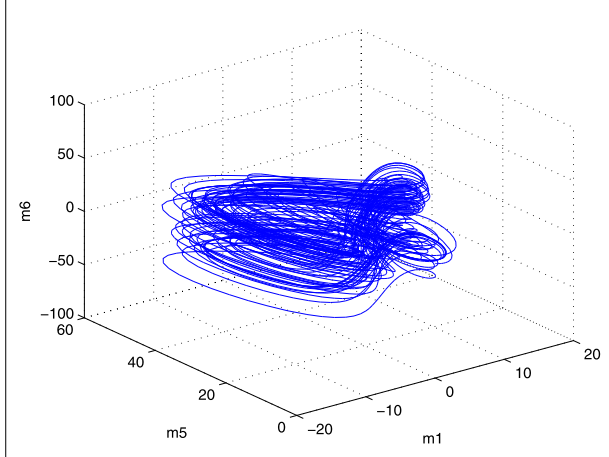

(c)

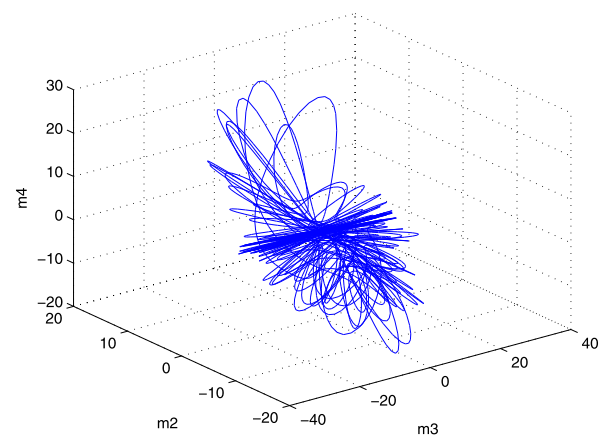

(b)

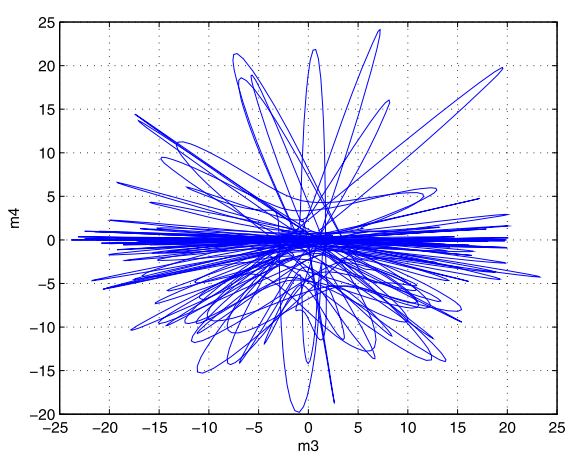

(d)

Figure 3 Hyperchaotic attractors of (4) in different phase and projections.

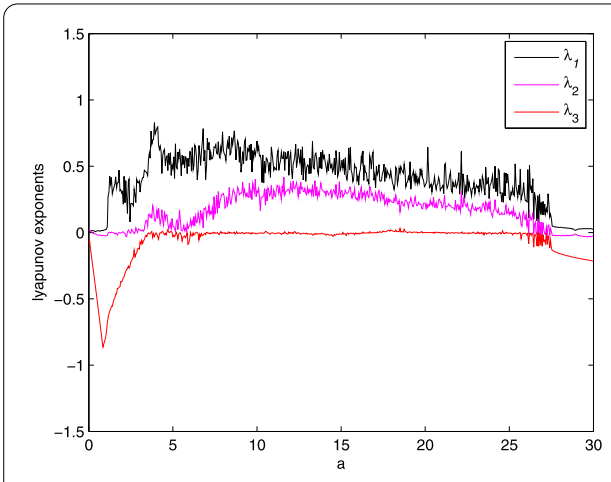

(a)

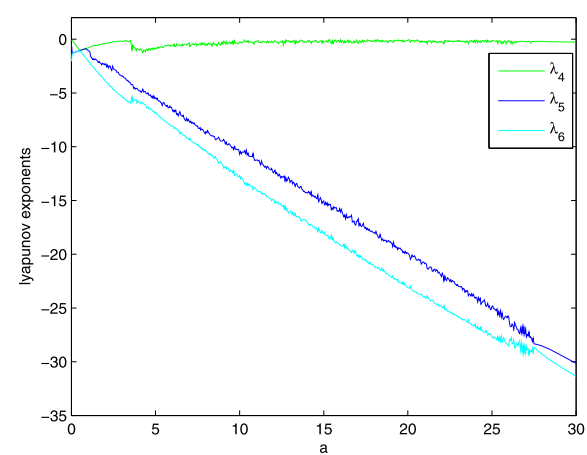

(b)

Figure 4 The Lyapunov exponents of system (4) with $a$ varying.

\subsection{Scheme of generalized combination complex synchronization}

Consider a $n_{1}$-dimensional chaotic complex system as the first drive system

$$
\dot{x}=A x+f(x),
$$

the second drive chaotic complex system with $n_{2}$ dimensions is given as

$$
\dot{y}=B y+g(y)
$$




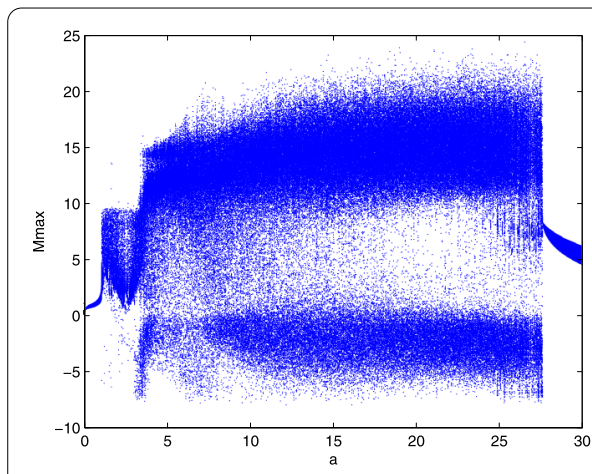

(a) Bifurcation diagram of system (4) with $a$ varying

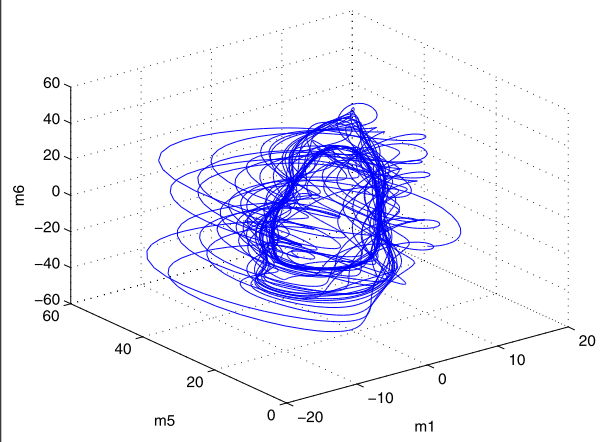

(c) $a=27$

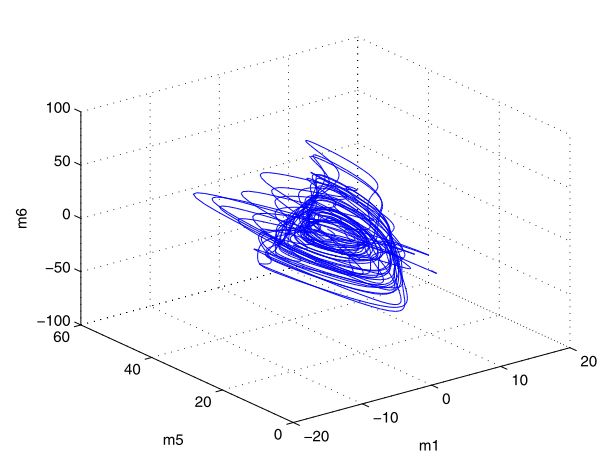

(b) $a=3$

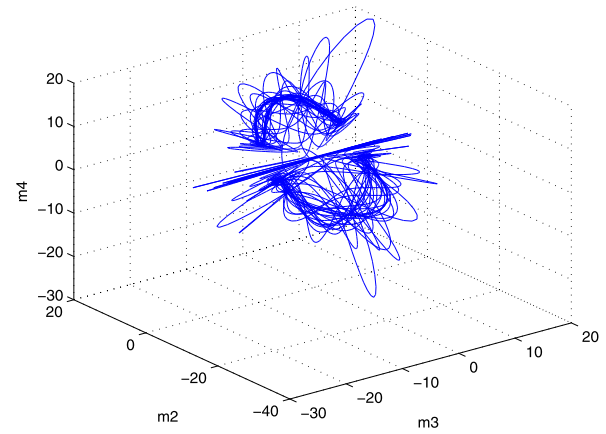

(d) $a=27$

Figure 5 Bifurcation diagram and attractors of system (4) with $a$ varying.

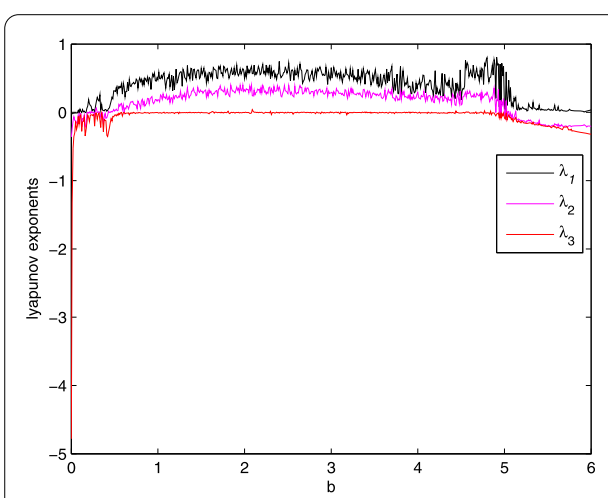

(a)

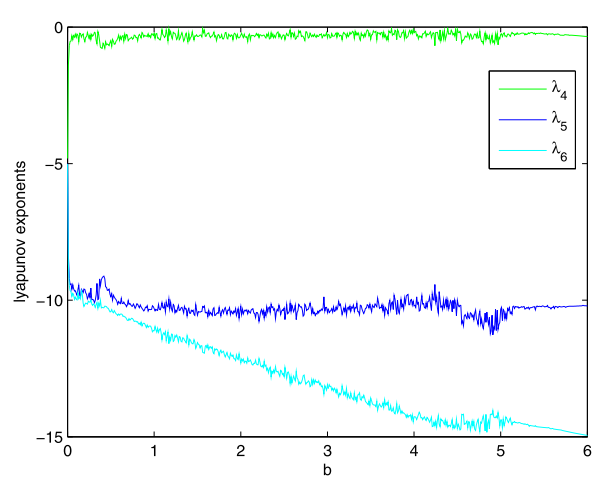

(b)

Figure 6 Lyapunov exponents of system (4) with $b$ varying.

while a $n$-dimensional response chaotic complex system is assumed to obey

$$
\dot{z}=C z+h(z)+U(x, y, z)
$$

where $x=x^{r}+j x^{i} \in \mathbb{C}^{n_{1} \times 1}, y=y^{r}+j y^{i} \in \mathbb{C}^{n_{2} \times 1}$, and $z=z^{r}+j z^{i} \in \mathbb{C}^{n \times 1}$ are the state complex vectors, $A \in \mathbb{R}^{n_{1} \times n_{1}}, B \in \mathbb{R}^{n_{2} \times n_{2}}$, and $C \in \mathbb{R}^{n \times n}$ are parameter matrices, while $f$, $g$, and $h$ are nonlinear complex functions and $U$ is a controller to be designed. 


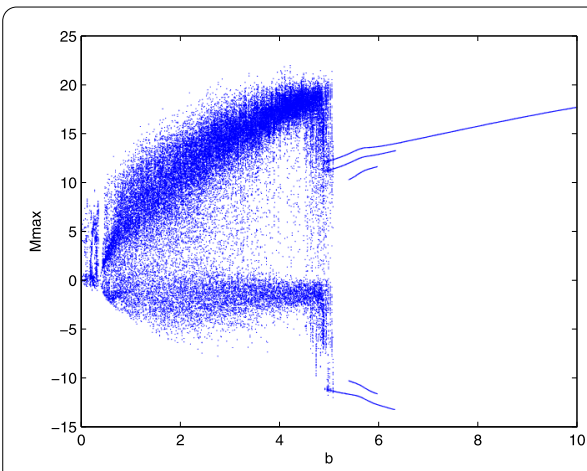

(a) Bifurcation diagram of system (4) with $b$ varying

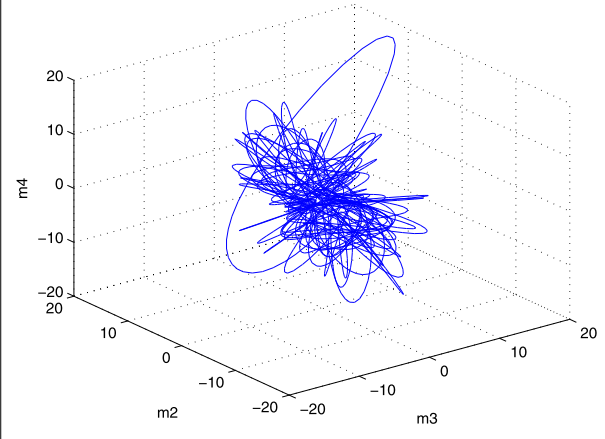

(c) $b=0.8$

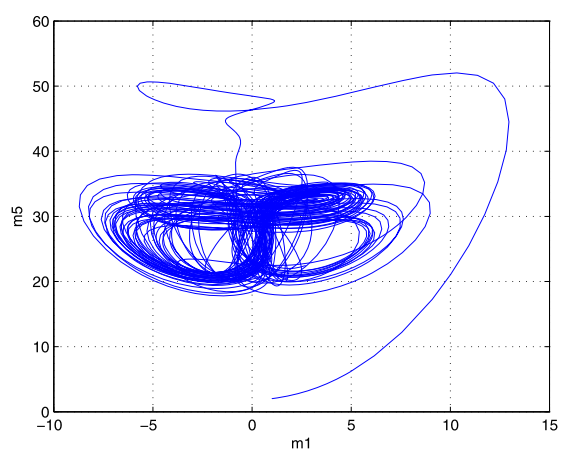

(b) $b=0.3$

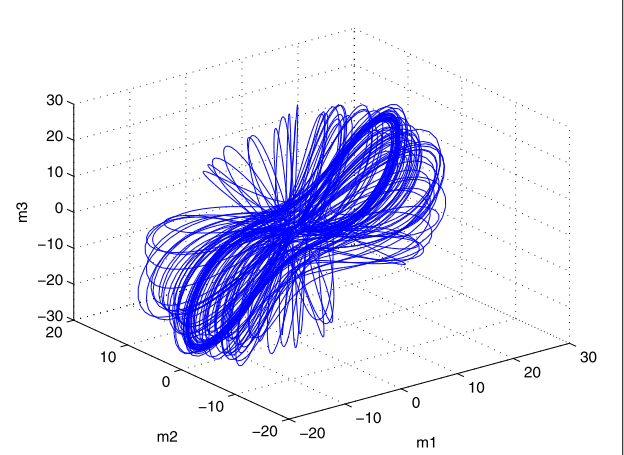

(d) $b=4.8$

Figure 7 Bifurcation diagram and attractors of system (4) with $b$ varying.

Remark 1 Many chaotic and hyperchaotic complex systems can be described by (5), such as the complex Lorenz system, the complex Chen system, the complex Lü system, the hyperchaotic complex Lorenz system, the hyperchaotic complex Lü system, etc.

The definition of generalized combination complex synchronization is introduced below.

Definition 1 For two drive systems (5), (6), and one response system (7), they are said to be in generalized combination complex synchronization if there exist two complex matrices $M_{1}=M_{1}^{r}+j M_{1}^{i} \in \mathbb{C}^{n \times n_{1}}$ and $M_{2}=M_{2}^{r}+j M_{2}^{i} \in \mathbb{C}^{n \times n_{2}}$, such that

$$
\lim _{t \rightarrow \infty}\|e\|=\lim _{t \rightarrow \infty}\left\|z-M_{1} x-M_{2} y\right\|=0
$$

where $\|\cdot\|$ is the matrix norm, $e=e^{r}+j e^{i}$ is called the error vector, $e^{r}=z^{r}-M_{1}^{r} x^{r}+M_{1}^{i} x^{i}-$ $M_{2}^{r} y^{r}+M_{2}^{i} y^{i}, e^{i}=z^{i}-M_{1}^{r} x^{i}-M_{1}^{i} x^{r}-M_{2}^{r} y^{i}-M_{2}^{i} y^{r}$, the complex matrices $M_{1}$ and $M_{2}$ are called the scaling matrices.

Remark 2 If the dimensions of the two drive systems (5) and (6) are equal to that of the response system (7), i.e., $n=n_{1}=n_{2}$, then the proposed synchronization will be combination complex synchronization.

Remark 3 If the scaling matrix $M_{1}=O_{n \times n_{1}}$ or $M_{2}=O_{n \times n_{2}}$, then we can achieve complex projective synchronization. If $M_{1}^{i}=O_{n \times n_{1}}$ or $M_{2}^{i}=O_{n \times n_{2}}$, then combination synchroniza- 
tion can be carried out. If $M_{1}=O_{n \times n_{1}}$ and $M_{2}=O_{n \times n_{2}}$, then the synchronization problem will be turned into a chaos control problem.

Remark 4 Definition 1 can be applicable to three or more chaotic complex systems. Additionally, drive systems and response systems can be identical or different.

The following lemma is useful in this paper.

Lemma 1 [25] For a matrix $D \in \mathbb{C}^{n \times n}$, all of the real parts of whose eigenvalues are negative, i.e., $\operatorname{Re}\left(\lambda_{i}(D)\right)<0(i=1,2, \ldots, n)$, then $\lim _{t \rightarrow \infty} \exp (D t)=0$.

Theorem 1 If the control law is chosen as follows:

$$
U=-C\left(M_{1} x+M_{2} y\right)-h(z)+M_{1}(A x+f(x))+M_{2}(B y+g(y))-K e,
$$

where $K$ is a complex control gain matrix, then generalized combination complex synchronization between the two drive systems (5), (6), and the response system (7) can be achieved if and only if all eigenvalues of $C-K$ satisfy $\operatorname{Re}\left(\lambda_{i}(C-K)\right)<0(i=1,2, \ldots, n)$.

Proof From Definition 1, we obtain the error vector between two drive systems (5), (6), and one response system (7) as follows:

$$
e(t)=z-M_{1} x-M_{2} y
$$

Calculating the derivative of the error vector (9) and with the designed controller (8), we conclude that

$$
\dot{e}(t)-(C-K) e(t)=0 .
$$

Multiplying $\exp ((-C-K) t)$ on both sides of (10), it follows that

$$
\frac{d(\exp (-(C-K) t) e(t))}{d t}=0 .
$$

Integrating equation (11) from 0 to $t$, it is straightforwardly found that

$$
e(t)=\exp ((C-K) t) e(0)
$$

where $e(0)$ is an initial condition. Thus, taking the limit on both sides of (12), since $\operatorname{Re}\left(\lambda_{i}(C-K)\right)<0$, by Lemma 1, we have

$$
\lim _{t \rightarrow \infty} e(t)=\lim _{t \rightarrow \infty} \exp ((C-K) t) e(0)=0 .
$$

Therefore, generalized combination complex synchronization is realized between the two drive systems (5), (6), and the response system (7) with the controller (8). This completes the proof.

In the following, we investigate generalized combination complex synchronization between real chaos and complex chaos. Now we consider two cases which include two real systems driving one complex system and two complex systems driving one real system. 
Corollary 1 Suppose that two drive systems (5) and (6) are chaotic real systems, i.e., $x \in$ $\mathbb{R}^{n_{1} \times 1}, y \in \mathbb{R}^{n_{2} \times 1}$, and the response system is a chaotic complex system (7), i.e., $z \in \mathbb{C}^{n \times 1}$. Then generalized combination complex synchronization between two drive real systems (5), (6), and one response complex system (7) can occur with the designed controller

$$
U=-C\left(M_{1} x+M_{2} y\right)-h(z)+M_{1}(A x+f(x))+M_{2}(B y+g(y))-K e,
$$

where $K \in \mathbb{C}^{n \times n}$, all eigenvalues of $C-K$ satisfy $\operatorname{Re}\left(\lambda_{i}(C-K)\right)<0(i=1,2, \ldots, n)$.

Corollary 2 Assume that two drive systems (5) and (6) are chaotic complex systems, i.e., $x \in \mathbb{C}^{n_{1} \times 1}, y \in \mathbb{C}^{n_{2} \times 1}$, and the response system (7) is a chaotic real system, where $z \in \mathbb{R}^{n \times 1}$. Since $z(t)$ is real, we choose a real controller $U$ to ensure combination synchronization of real parts and avoid increasing the imaginary parts of the response system. Consequently, the error vector is defined as

$$
e=z-M_{1}^{r} x^{r}+M_{1}^{i} x^{i}-M_{2}^{r} y^{r}+M_{2}^{i} y^{i}
$$

If the real controller is designed as

$$
\begin{aligned}
U= & -h(z)-C\left(M_{1}^{r} x^{r}-M_{1}^{i} x^{i}+M_{2}^{r} y^{r}-M_{2}^{i} y^{i}\right)+M_{1}^{r}\left(A x^{r}+f^{r}(x)\right)-M_{1}^{i}\left(A x^{i}+f^{i}(x)\right) \\
& +M_{2}^{r}\left(B y^{r}+g^{r}(y)\right)-M_{2}^{i}\left(B y^{i}+g^{i}(y)\right)-K e
\end{aligned}
$$

where $K \in \mathbb{R}^{n \times n}$, all eigenvalues of $C-K$ satisfy $\operatorname{Re}\left(\lambda_{i}(C-K)\right)<0(i=1,2, \ldots, n)$, then the two drive complex systems (5), (6), and the response real system (7) are in generalized combination complex synchronization of real parts.

In addition, from Theorem 1, some corollaries can easily be obtained and their proofs are omitted.

Corollary 3 (I) Suppose $M_{1}=O_{n \times n_{1}}$. If the controller is designed as follows:

$$
U=-C M_{2} y-h(z)+M_{2}(B y+g(y))-K e,
$$

where $K$ is a control gain matrix, then complex projective synchronization between two different dimensional systems (6) and (7) can be realized if and only if all eigenvalues of $C-K$ satisfy $\operatorname{Re}\left(\lambda_{i}(C-K)\right)<0(i=1,2, \ldots, n)$.

(II) Suppose $M_{2}=O_{n \times n_{2}}$. If the controller is designed as follows:

$$
U=-C M_{1} x-h(z)+M_{1}(A x+f(x))-K e,
$$

where $K$ is a control gain matrix, then complex projective synchronization between two different dimensional systems (5) and (7) can occur if and only if all eigenvalues of $C-K$ satisfy $\operatorname{Re}\left(\lambda_{i}(C-K)\right)<0(i=1,2, \ldots, n)$.

Corollary 4 Assume two scaling matrices $M_{1}=O_{n \times n_{1}}$ and $M_{2}=O_{n \times n_{2}}$. If the complex controller is given as

$$
U=-h(z)-K e,
$$


where $K$ is a control gain matrix and all eigenvalues of $C-K$ satisfy $\operatorname{Re}\left(\lambda_{i}(C-K)\right)<0$ $(i=1,2, \ldots, n)$, then the equilibrium point of the response system (7) is asymptotically stable.

\subsection{Numerical examples}

In this subsection, we provide two examples to illustrate the feasibility and effectiveness of the proposed schemes. Firstly, synchronization between two 3-dimensional chaotic complex systems and a 4-dimensional new hyperchaotic complex system is studied.

\subsubsection{Synchronization between two drive chaotic complex systems and a response hyperchaotic complex system}

Now, we consider that the complex Lü system and the complex Lorenz system drive a hyperchaotic complex Lü-like system. Thus, the two drive systems are given as

$$
\begin{aligned}
& \left\{\begin{array}{l}
\dot{x}_{1}=a_{1}\left(x_{2}-x_{1}\right), \\
\dot{x}_{2}=-x_{1} x_{3}+a_{2} x_{2}, \\
\dot{x}_{3}=\frac{1}{2}\left(\bar{x}_{1} x_{2}+x_{1} \bar{x}_{2}\right)-a_{3} x_{3},
\end{array}\right. \\
& \left\{\begin{array}{l}
\dot{y}_{1}=b_{1}\left(y_{2}-y_{1}\right) \\
\dot{y}_{2}=b_{2} y_{1}-y_{2}-y_{1} y_{3} \\
\dot{y}_{3}=\frac{1}{2}\left(\bar{y}_{1} y_{2}+y_{1} \bar{y}_{2}\right)-b_{3} y_{3},
\end{array}\right.
\end{aligned}
$$

where

$$
\begin{array}{ll}
A=\left(\begin{array}{ccc}
-a_{1} & a_{1} & 0 \\
0 & a_{2} & 0 \\
0 & 0 & -a_{3}
\end{array}\right), & f(x)=\left(\begin{array}{c}
0 \\
-x_{1} x_{3} \\
\frac{1}{2}\left(\bar{x}_{1} x_{2}+x_{1} \bar{x}_{2}\right)
\end{array}\right), \\
B=\left(\begin{array}{ccc}
-b_{1} & b_{1} & 0 \\
b_{2} & -1 & 0 \\
0 & 0 & -b_{3}
\end{array}\right), & g(y)=\left(\begin{array}{c}
0 \\
-y_{1} y_{3} \\
\frac{1}{2}\left(\bar{y}_{1} y_{2}+y_{1} \bar{y}_{2}\right)
\end{array}\right) ;
\end{array}
$$

$x_{1}=x_{1}^{r}+j x_{1}^{i}, x_{2}=x_{2}^{r}+j x_{2}^{i}, y_{1}=y_{1}^{r}+j y_{1}^{i}$ and $y_{2}=y_{2}^{r}+j y_{2}^{i}$ are complex variables, $x_{3}$ and $y_{3}$ are real variables. Systems (13) and (14) behave chaotically with the given parameters $\left(a_{1}, a_{2}, a_{3}\right)=(40,22,5)$ and $\left(b_{1}, b_{2}, b_{3}\right)=(14,35,3.7)$, respectively; see Figure 8 .

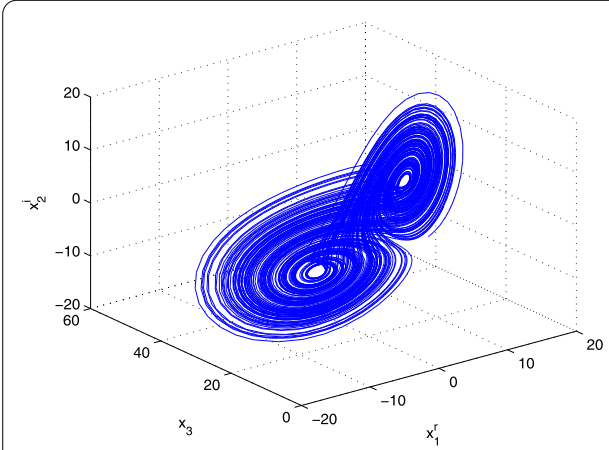

(a) Attractor of the complex Lü system

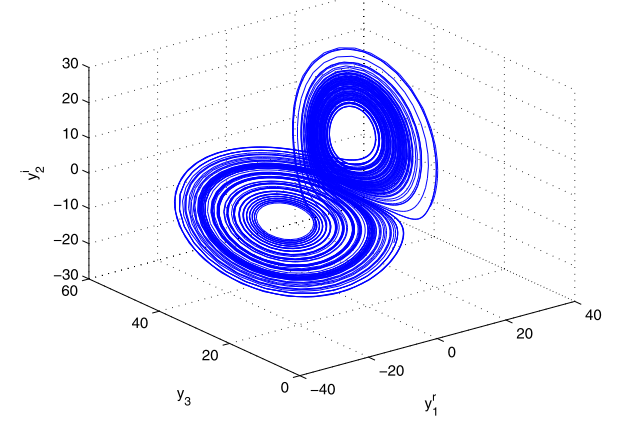

(b) Attractor of the complex Lorenz system

Figure 8 Chaotic attractors of systems (13) and (14). 
The response system is the proposed hyperchaotic complex Lü-like system:

$$
\left\{\begin{array}{l}
\dot{z}_{1}=c_{1}\left(z_{2}-z_{1}\right)+U_{1} \\
\dot{z}_{2}=c_{2} z_{1}-z_{1} z_{3}+z_{4}+U_{2} \\
\dot{z}_{3}=\frac{1}{2}\left(\bar{z}_{1} z_{2}+z_{1} \bar{z}_{2}\right)-c_{3} z_{3}+U_{3} \\
\dot{z}_{4}=-\frac{c_{4}}{2}\left(\bar{z}_{2}+z_{2}\right)+U_{4}
\end{array}\right.
$$

where

$$
C=\left(\begin{array}{cccc}
-c_{1} & c_{1} & 0 & 0 \\
c_{2} & 0 & 0 & 1 \\
0 & 0 & -c_{3} & 0 \\
0 & -\frac{c_{4}}{2} & 0 & 0
\end{array}\right), \quad h(z)=\left(\begin{array}{c}
0 \\
-z_{1} z_{3} \\
\frac{1}{2}\left(\bar{z}_{1} z_{2}+z_{1} \bar{z}_{2}\right) \\
-\frac{c_{4}}{2} \bar{z}_{2}
\end{array}\right)
$$

$z_{1}=z_{1}^{r}+j z_{1}^{i}$ and $z_{2}=z_{2}^{r}+j z_{2}^{i}$ are complex variables, $z_{3}$ and $z_{4}$ are real variables. $U=$ $\left(U_{1}, U_{2}, U_{3}, U_{4}\right)^{T}$ is a controller to be determined.

Here, we select two complex scaling matrices $M_{1}$ and $M_{2}$ as

$$
M_{1}=\left(\begin{array}{ccc}
j & 1 & -2 \\
1 & -j & 1 \\
0 & 0 & -2 \\
0 & 0 & 1
\end{array}\right), \quad M_{2}=\left(\begin{array}{ccc}
-j & 1 & -1 \\
-4 & j & 1 \\
0 & 0 & 1 \\
0 & 0 & 2
\end{array}\right)
$$

thus the error system $e=z-M_{1} x-M_{2} y$ is obtained as follows:

$$
\left\{\begin{array}{l}
e_{1}=z_{1}-x_{2}+2 x_{3}-y_{2}+y_{3}+j\left(y_{1}-x_{1}\right) \\
e_{2}=z_{2}-x_{1}-x_{3}+4 y_{1}-y_{3}+j\left(x_{2}-y_{2}\right) \\
e_{3}=z_{3}+2 x_{3}-y_{3} \\
e_{4}=z_{4}-x_{3}-2 y_{3}
\end{array}\right.
$$

A control matrix is chosen as

$$
K=\left(\begin{array}{cccc}
-7 & 6 & -1-j & 0 \\
29 & 3 & 0 & -1-j \\
0 & 0 & -2 / 3 & 0 \\
0 & -5 & -3 & 1
\end{array}\right)
$$

and with the choice of $\left(c_{1}, c_{2}, c_{3}, c_{4}\right)=(10,28,8 / 3,10)$ we have

$$
C-K=\left(\begin{array}{cccc}
-3 & 4 & 1+j & 0 \\
-1 & -3 & 0 & 2+j \\
0 & 0 & -2 & 0 \\
0 & 0 & 3 & -1
\end{array}\right)
$$

After a routine calculation, we obtain the eigenvalues of $C-K$ are $\lambda_{1}=-3+2 j, \lambda_{2}=-3-2 j$, $\lambda_{3}=-2$, and $\lambda_{4}=-1$. It is clear that all eigenvalues of $C-K$ satisfy $\operatorname{Re}\left(\lambda_{i}(C-K)\right)<0$ $(i=1,2,3,4)$. 


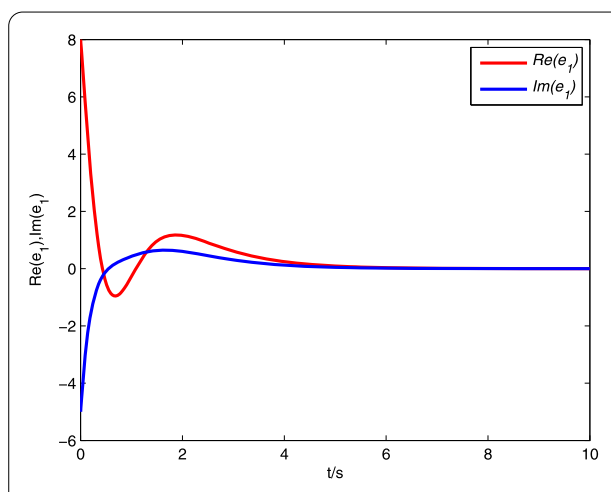

(a)

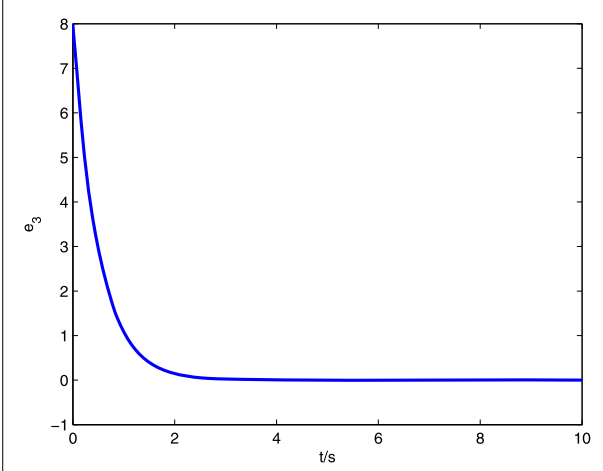

(c)

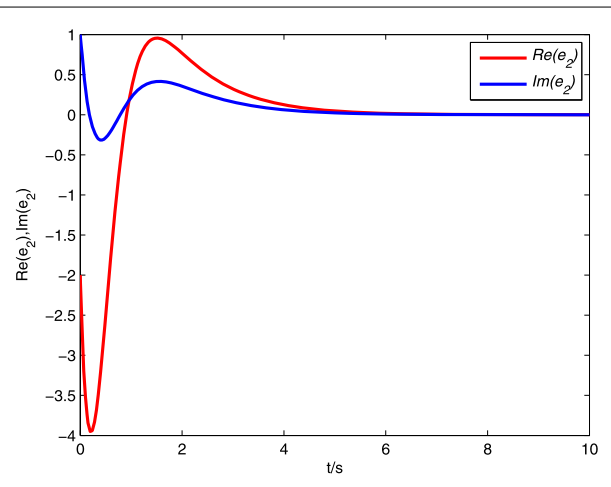

(b)

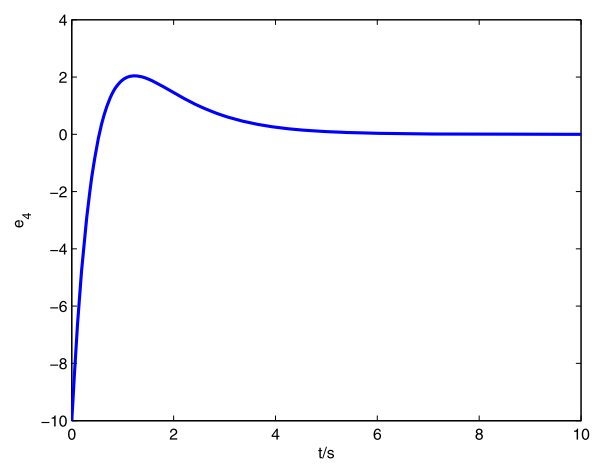

(d)

Figure 9 The time evolution of synchronization errors between systems (13), (14), and (15).

According to Theorem 1, a complex controller is designed as follows:

$$
\left\{\begin{aligned}
U_{1}= & \left(a_{2}+c_{1}\right) x_{2}+\left(c_{1}-1\right) y_{2}+\left(2 a_{3}-3 c_{1}\right) x_{3}+\left(b_{3}-2 c_{1}\right) y_{3}-c_{1} x_{1} \\
& +\left(b_{2}+4 c_{1}\right) y_{1}-x_{1} x_{3}-2 x_{1}^{r} x_{2}^{r}-2 x_{1}^{i} x_{2}^{i}-y_{1} y_{3}-y_{1}^{r} y_{2}^{r}-y_{1}^{i} y_{2}^{i}+7 e_{1}-6 e_{2} \\
& +(1+j) e_{3}+j\left(\left(c_{1}-a_{1}\right) x_{1}+\left(b_{1}-c_{1}\right) y_{1}-\left(b_{1}+c_{1}\right) y_{2}+\left(a_{1}+c_{1}\right) x_{2}\right), \\
U_{2}= & \left(a_{1}-c_{2}\right) x_{2}-\left(c_{2}+4 b_{1}\right) y_{2}+\left(2 c_{2}-1-a_{3}\right) x_{3}+\left(c_{2}-2-b_{3}\right) y_{3}-a_{1} x_{1} \\
& +4 b_{1} y_{1}+z_{1} z_{3}+x_{1}^{r} x_{2}^{r}+x_{1}^{i} x_{2}^{i}+y_{1}^{r} y_{2}^{r}+y_{1}^{i} y_{2}^{i}-29 e_{1}-3 e_{2}+(1+j) e_{4} \\
& +j\left(x_{1} x_{3}-y_{1} y_{3}+\left(b_{2}+c_{2}\right) y_{1}-c_{2} x_{1}-y_{2}-a_{2} x_{2}\right), \\
U_{3}= & 2\left(a_{3}-c_{3}\right) x_{3}+\left(c_{3}-b_{3}\right) y_{3}-z_{1}^{r} z_{2}^{r}-z_{1}^{i} z_{2}^{i}-2 x_{1}^{r} x_{2}^{r}-2 x_{1}^{i} x_{2}^{i}+y_{1}^{r} y_{2}^{r}+y_{1}^{i} y_{2}^{i}+\frac{2}{3} e_{3}, \\
U_{4}= & 10 z_{2}^{r}-a_{3} x_{3}-2 b_{3} y_{3}+x_{1}^{r} x_{2}^{r}+x_{1}^{i} x_{2}^{i}+y_{1}^{r} y_{2}^{r}+y_{1}^{i} y_{2}^{i}+3 e_{3}-e_{4} .
\end{aligned}\right.
$$

In the numerical simulations, the initial values of the drive and response systems are chosen as $x(0)=(1+2 j, 3+4 j, 5)^{T}, y(0)=(2+j, 5+3 j, 4)^{T}$, and $z(0)=(1+j, 1+j, 2,3)^{T}$, respectively. Figure 9 displays that the errors of synchronization tend to zero, i.e., synchronization between two drive chaotic complex systems and a response hyperchaotic complex system is realized.

\subsubsection{Synchronization between two drive hyperchaotic complex systems and a response chaotic real system}

Next, we investigate synchronization between two 4-dimensional hyperchaotic complex systems and a 3-dimensional chaotic real system. Assume that two hyperchaotic complex 
Lü-like systems drive the real Lorenz system [26]. Thus, two drive systems are described as follows:

$$
\begin{aligned}
& \left\{\begin{array}{l}
\dot{x}_{1}=a_{1}\left(x_{2}-x_{1}\right), \\
\dot{x}_{2}=a_{2} x_{1}-x_{1} x_{3}+x_{4}, \\
\dot{x}_{3}=\frac{1}{2}\left(\bar{x}_{1} x_{2}+x_{1} \bar{x}_{2}\right)-a_{3} x_{3}, \\
\dot{x}_{4}=-\frac{a_{4}}{2}\left(\bar{x}_{2}+x_{2}\right),
\end{array}\right. \\
& \left\{\begin{array}{l}
\dot{y}_{1}=b_{1}\left(y_{2}-y_{1}\right), \\
\dot{y}_{2}=b_{2} y_{1}-y_{1} y_{3}+y_{4}, \\
\dot{y}_{3}=\frac{1}{2}\left(\bar{y}_{1} y_{2}+y_{1} \bar{y}_{2}\right)-b_{3} y_{3}, \\
\dot{y}_{4}=-\frac{b_{4}}{2}\left(\bar{y}_{2}+y_{2}\right),
\end{array}\right.
\end{aligned}
$$

where

$$
\begin{aligned}
A & =\left(\begin{array}{cccc}
-a_{1} & a_{1} & 0 & 0 \\
a_{2} & 0 & 0 & 1 \\
0 & 0 & -a_{3} & 0 \\
0 & -\frac{a_{4}}{2} & 0 & 0
\end{array}\right), & f(x)=\left(\begin{array}{c}
0 \\
-x_{1} x_{3} \\
\frac{1}{2}\left(\bar{x}_{1} x_{2}+x_{1} \bar{x}_{2}\right) \\
-\frac{a_{4}}{2} \bar{x}_{2}
\end{array}\right), \\
B & =\left(\begin{array}{cccc}
-b_{1} & b_{1} & 0 & 0 \\
b_{2} & 0 & 0 & 1 \\
0 & 0 & -b_{3} & 0 \\
0 & -\frac{b_{4}}{2} & 0 & 0
\end{array}\right), & g(y)=\left(\begin{array}{c}
0 \\
-y_{1} y_{3} \\
\frac{1}{2}\left(\bar{y}_{1} y_{2}+y_{1} \bar{y}_{2}\right) \\
-\frac{b_{4}}{2} \bar{y}_{2}
\end{array}\right) ;
\end{aligned}
$$

$x_{1}=x_{1}^{r}+j x_{1}^{i}, x_{2}=x_{2}^{r}+j x_{2}^{i}, y_{1}=y_{1}^{r}+j y_{1}^{i}$ and $y_{2}=y_{2}^{r}+j y_{2}^{i}$ are complex variables, $x_{3}, x_{4}, y_{3}$, and $y_{4}$ are real variables. Suppose $\left(a_{1}, a_{2}, a_{3}, a_{4}\right)=\left(b_{1}, b_{2}, b_{3}, b_{4}\right)=(10,28,8 / 3,10), x(0)=$ $(1+j, 1+j, 2,3)^{T}$, and $y(0)=(-2+j, 4-5 j, 10,3)^{T}$. Systems (16) and (17) are hyperchaotic; see Figure 3 and Figure 10(a), respectively.

The real Lorenz system reads

$$
\left\{\begin{array}{l}
\dot{z}_{1}=c_{1}\left(z_{2}-z_{1}\right)+U_{1}, \\
\dot{z}_{2}=c_{2} z_{1}-z_{1} z_{3}-z_{2}+U_{2}, \\
\dot{z}_{3}=z_{1} z_{2}-c_{3} z_{3}+U_{3},
\end{array}\right.
$$

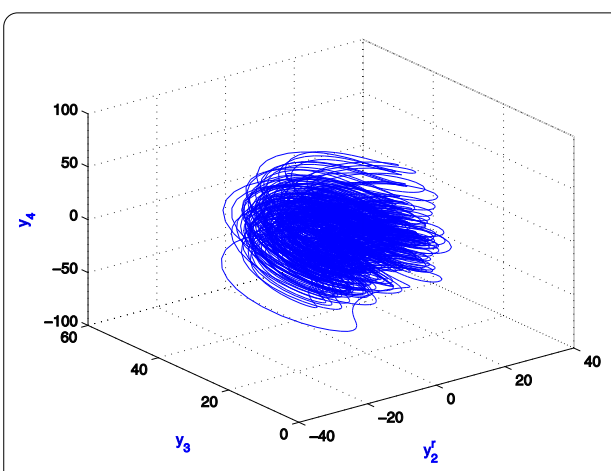

(a) Attractor of hyperchaotic complex Lü-like system

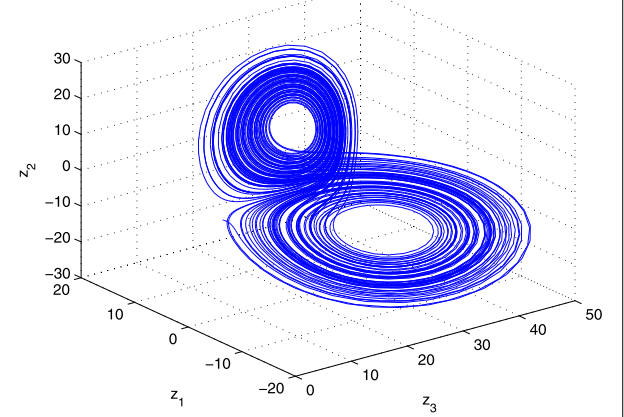

(b) Attractor of the real Lorenz system

Figure 10 Attractors of hyperchaotic system (17) and chaotic system (18). 
where

$$
C=\left(\begin{array}{ccc}
-c_{1} & c_{1} & 0 \\
c_{2} & -1 & 0 \\
0 & 0 & -c_{3}
\end{array}\right), \quad h(z)=\left(\begin{array}{c}
0 \\
-z_{1} z_{3} \\
z_{1} z_{2}
\end{array}\right)
$$

$z_{1}, z_{2}$, and $z_{3}$ are real variables, $U=\left(U_{1}, U_{2}, U_{3}\right)^{T}$ is a real controller which will be designed later. For the choice of $\left(c_{1}, c_{2}, c_{3}\right)=(10,28,8 / 3)$, system (18) is chaotic as shown in Figure 10(b).

Assume two complex scaling matrices $M_{1}$ and $M_{2}$ as follows:

$$
\begin{aligned}
& M_{1}=\left(\begin{array}{cccc}
j & 1 & -j & 2 j \\
2 & j & 4 j & -j \\
1 & 0 & 1+j & j
\end{array}\right)=\left(\begin{array}{cccc}
0 & 1 & 0 & 0 \\
2 & 0 & 0 & 0 \\
1 & 0 & 1 & 0
\end{array}\right)+j\left(\begin{array}{cccc}
1 & 0 & -1 & 2 \\
0 & 1 & 4 & -1 \\
0 & 0 & 1 & 1
\end{array}\right), \\
& M_{2}=\left(\begin{array}{cccc}
1 & j & 3 j & j \\
j & -2 & j & 2 j \\
0 & 1 & j & 1+j
\end{array}\right)=\left(\begin{array}{cccc}
1 & 0 & 0 & 0 \\
0 & -2 & 0 & 0 \\
0 & 1 & 0 & 1
\end{array}\right)+j\left(\begin{array}{llll}
0 & 1 & 3 & 1 \\
1 & 0 & 1 & 2 \\
0 & 0 & 1 & 1
\end{array}\right) .
\end{aligned}
$$

Thus the real error system $e=z-M_{1}^{r} x^{r}+M_{1}^{i} x^{i}-M_{2}^{r} y^{r}+M_{2}^{i} y^{i}$ is written in the following form:

$$
\left\{\begin{array}{l}
e_{1}=z_{1}-x_{2}^{r}+x_{1}^{i}-y_{1}^{r}+y_{2}^{i} \\
e_{2}=z_{2}-2 x_{1}^{r}+x_{2}^{i}+2 y_{2}^{r}+y_{1}^{i} \\
e_{3}=z_{3}-x_{1}^{r}-x_{3}-y_{2}^{r}-y_{4}
\end{array}\right.
$$

A real control matrix is chosen as

$$
K=\left(\begin{array}{ccc}
-5 & 9 & 0 \\
29 & 4 & 0 \\
0 & 0 & -5 / 3
\end{array}\right)
$$

then we have

$$
C-K=\left(\begin{array}{ccc}
-5 & 1 & 0 \\
-1 & -5 & 0 \\
0 & 0 & -1
\end{array}\right)
$$

for the case of $\left(c_{1}, c_{2}, c_{3}\right)=(10,28,8 / 3)$.

According to Corollary 2, the real controller is designed as

$$
\left\{\begin{aligned}
U_{1}= & \left(a_{1}-c_{1}\right)\left(x_{1}^{i}-x_{2}^{i}\right)-\left(b_{1}-c_{1}\right) y_{1}^{r}+\left(a_{2}-2 c_{1}\right) x_{1}^{r}+\left(b_{1}+2 c_{1}\right) y_{2}^{r} \\
& +\left(c_{1}-b_{2}\right) y_{1}^{i}+c_{1}\left(x_{2}^{r}-y_{2}^{i}\right)-x_{1}^{r} x_{3}+x_{4}+y_{1}^{i} y_{3}+5 e_{1}-9 e_{2}, \\
U_{2}= & z_{1} z_{3}+\left(2 a_{1}-c_{2}\right) x_{2}^{r}+\left(c_{2}-a_{2}\right) x_{1}^{i}-\left(2 b_{2}+c_{2}\right) y_{1}^{r}+\left(c_{2}-b_{1}\right) y_{2}^{i} \\
& +2\left(1-a_{1}\right) x_{1}^{r}-x_{2}^{i}-2 y_{2}^{r}+x_{1}^{i} x_{3}+\left(b_{1}-1\right) y_{1}^{i}+2 y_{1}^{r} y_{3}-2 y_{4}-29 e_{1}-4 e_{2}, \\
U_{3}= & \left(c_{3}-a_{1}\right) x_{1}^{r}-z_{1} z_{2}+\left(c_{3}-a_{3}\right) x_{3}+\left(c_{3}-b_{4}\right) y_{2}^{r}+\left(c_{3}+1\right) y_{4}+a_{1} x_{2}^{r} \\
& +x_{1}^{r} x_{2}^{r}+x_{1}^{i} x_{2}^{i}+b_{2} y_{1}^{r}-y_{1}^{r} y_{3}+\frac{5}{3} e_{3} .
\end{aligned}\right.
$$




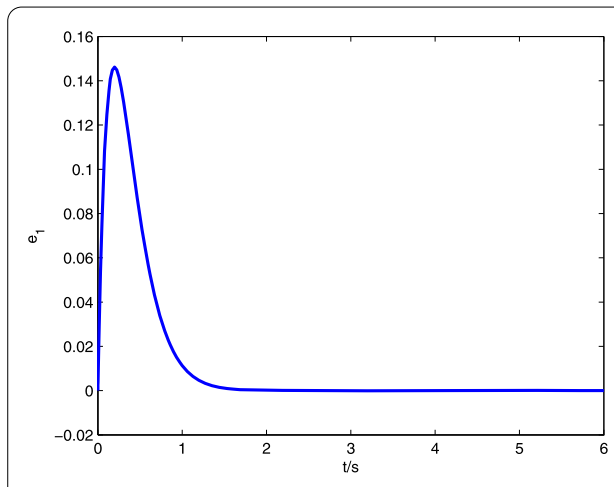

(a)

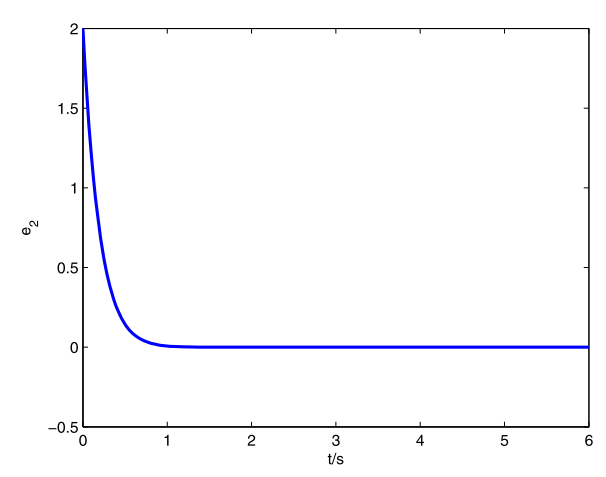

(b)

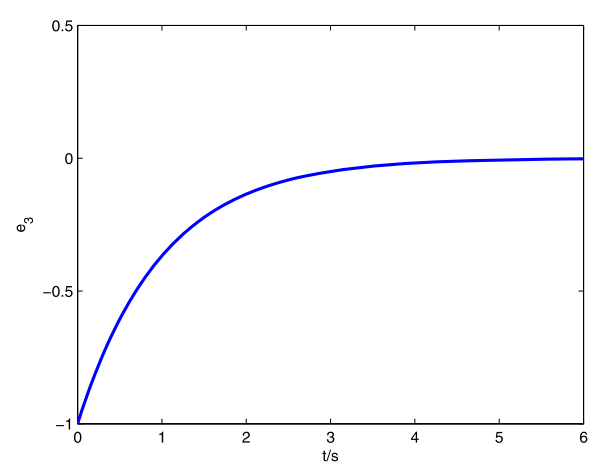

(c)

Figure 11 The time evolution of synchronization errors between systems (16), (17), and (18).

In the numerical simulations, the initial values of the drive and response systems are $x(0)=(1+j, 1+j, 2,3)^{T}, y(0)=(-2+j, 4-5 j, 10,3)^{T}$, and $z(0)=(3,-6,9)^{T}$, respectively. All eigenvalues of $C-K$ are $\lambda_{1}=-5+j, \lambda_{2}=-5-j$, and $\lambda_{3}=-1$, which satisfy $\operatorname{Re}\left(\lambda_{i}(C-K)\right)<0$ $(i=1,2,3)$. The errors of synchronization converge asymptotically to zero, which is shown in Figure 11. Therefore, synchronization between two drive hyperchaotic complex systems and a response chaotic real system is achieved in the real parts.

\section{Conclusions}

In this work, we firstly introduce a new hyperchaotic complex system and study its dynamical behavior. The dynamical properties of this new system are identified by using phase portraits, bifurcation diagrams, and the Lyapunov exponents spectra. Secondly, we propose generalized combination synchronization between three different dimensional chaotic complex systems. In this proposed scheme, two drive systems and one response system can be synchronized to two complex scaling matrices which are non-square matrices. Besides, a general controller is designed to achieve generalized combination complex synchronization. Through this scheme, generalized combination synchronization between real chaos and complex chaos can be investigated by virtue of two complex scaling matrices. It is worth mentioning that there are various types of synchronization are special cases from our definition, which are complex projective synchronization, combination synchronization, and combination complex synchronization. Therefore, the obtained results extend many existing results in the literature. Moreover, many problems with un- 
known parameters and external disturbances exist in practical chaotic synchronization. Consequently, we will make an endeavor to investigate robust generalized combination complex synchronization considering unknown parameters and external disturbances in our future work.

\section{Competing interests}

The authors declare that they have no competing interests.

\section{Authors' contributions}

The two authors contributed equally to this work. They all read and approved the final version of the manuscript.

\section{Acknowledgements}

The authors would like to thank the editors and anonymous referees for their constructive comments and suggestions. The research was supported by the National Natural Science Foundation of China (numbers 61273088, 10971120) and the Natural Science Foundation of Shandong Province (number ZR2010FM010).

Received: 17 November 2014 Accepted: 29 April 2015 Published online: 11 July 2015

\section{References}

1. Fowler, AC, Gibbon, JD, McGuinness, MJ: The complex Lorenz equations. Physica D 4, 139-163 (1982)

2. Gao, TG, Chen, ZQ: A new image encryption algorithm based on hyper-chaos. Phys. Lett. A 372, 394-400 (2008)

3. Mahmoud, GM, Mahmoud, EE, Arafa, AA: On projective synchronization of hyperchaotic complex nonlinear systems based on passive theory for secure communications. Phys. Scr. 87, 055002 (2013)

4. Liu, ST, Zhang, FF: Complex function projective synchronization of complex chaotic system and its applications in secure communications. Nonlinear Dyn. 76, 1087-1097 (2014)

5. Mahmoud, GM, Al-Kashif, MA, Aly, SA: Basic properties and chaotic synchronization of complex Lorenz system. Int. J. Mod. Phys. C 18, 253-265 (2007)

6. Mahmoud, GM, Bountis, T, Mahmoud, EE: Active control and global synchronization of the complex Chen and Lü systems. Int. J. Bifurc. Chaos 17, 4295-4308 (2007)

7. Mahmoud, GM, Ahmed, ME, Mahmoud, EE: Analysis of hyperchaotic complex Lorenz systems. Int. J. Mod. Phys. C 19, 1477-1494 (2008)

8. Mahmoud, GM, Mahmoud, EE, Ahmed, ME: On the hyperchaotic complex Lü systems. Nonlinear Dyn. 58, 725-738 (2009)

9. Mahmoud, GM, Mahmoud, EE: Complete synchronization of chaotic complex nonlinear systems with uncertain parameters. Nonlinear Dyn. 62, 875-882 (2010)

10. Liu, P, Liu, ST: Anti-synchronization of chaotic complex nonlinear systems. Phys. Scr. 83, 065006 (2011)

11. Liu, P, Liu, ST: Adaptive anti-synchronization of chaotic complex nonlinear systems with unknown parameters. Nonlinear Anal., Real World Appl. 12, 3046-3055 (2010)

12. Mahmoud, GM, Mahmoud, EE: Synchronization and control of hyperchaotic complex Lorenz systems. Math. Comput. Simul. 80, 2286-2296 (2010)

13. Mahmoud, EE: Complex complete synchronization of two non-identical hyperchaotic complex nonlinear systems. Math. Methods Appl. Sci. 37, 321-328 (2014)

14. Wu, ZY, Duan, JQ, Fu, XC: Complex projective synchronization in coupled chaotic complex dynamical systems. Nonlinear Dyn. 69, 771-779 (2012)

15. Zhang, FF, Liu, ST: Full state hybrid projective synchronization and parameters identification for uncertain chaotic (hyperchaotic) complex systems. J. Comput. Nonlinear Dyn. 9, 021009 (2013)

16. Mahmoud, GM, Mahmoud, EE: Complex modified projective synchronization of two chaotic complex nonlinear systems. Nonlinear Dyn. 73, 2231-2240 (2013)

17. Luo, RZ, Wang, YL, Deng, SC: Combination synchronization of three classic chaotic systems using active backstepping design. Chaos 21, e043114 (2011)

18. $\mathrm{Wu}, \mathrm{ZY}, \mathrm{Fu}, \mathrm{XC}$ : Combination synchronization of three different order nonlinear systems using active backstepping design. Nonlinear Dyn. 73, 1863-1872 (2013)

19. Zhou, $X B$, Jiang, MR, Huang, YQ: Combination synchronization of three identical or different nonlinear complex hyperchaotic systems. Entropy 15, 3746-3761 (2013)

20. Sun, JW, Cui, GZ, Wang, YF, Shen, Y: Combination complex synchronization of three chaotic complex systems. Nonlinear Dyn. 79, 953-963 (2015)

21. Bazhenov, M, Huerta, R, Rabinovich, MI, Sejnowski, T: Cooperative behavior of a chain of synaptically coupled chaotic neurons. Physica D 116, 392-400 (1998)

22. Zhou, WN, Xu, YH, Lu, HQ, Pan, L: On dynamic analysis of a new chaotic attractor. Phys. Lett. A 372, 5773-5777 (2008)

23. Alazzawi, SF: Study of dynamical properties and effective of a state $u$ for hyperchaotic Pan systems. Al-Rafiden J. Comput. Sci. Math. 10, 89-99 (2013)

24. Frederickson, P, Kaplan, JL, Yorke, JA: The Lyapunov dimension of strange attractors. J. Differ. Equ. 44, $185-207$ (1983)

25. Wei, HC, Zheng, XC: The Matrix Theory in Engineering. China University of Petroleum Press, Dongying (1999)

26. Lorenz, EN: Deterministic nonperiodic flow. J. Atmos. Sci. 20, 130-141 (1963) 